\title{
Effets de la microdose sur la production du niébé, du mil et du sorgho en fonction la toposéquence
}

\author{
Fatimata SABA*, Sibiri Jean Baptiste TAONDA, Idriss SERME, \\ Alimata A. BANDAOGO, Augustin P SOURWEMA et Adama KABRE \\ Institut de l'Environnement et de Recherches Agricoles (INERA), Burkina Faso. \\ *Auteur correspondant ; E-mail : fati.saba@yahoo.fr
}

\section{RESUME}

La baisse de la fertilité des sols agit fortement sur la productivité des cultures et par conséquent, le revenu des agriculteurs. L'utilisation des engrais minéraux permet d'améliorer la productivité des sols mais son accès reste limité aux petits producteurs. L'objectif de la présente étude est d'évaluer l'efficacité agronomique et économique de la microdose selon la toposéquence. Des tests ont été implantés suivant la toposéquence pendant deux ans et des enquêtes ont été menées auprès des ménages pour évaluer la rentabilité de la technologie. Des apports de NPK (14-23-14) en microdose à raison de 2 g/poquet ont été effectués sur le niébé et le sorgho et $3 \mathrm{~g} /$ poquet au mil (soit $62,5 \mathrm{~kg} / \mathrm{ha}$ ). Le sorgho a reçu en plus $1 \mathrm{~g}$ d'urée par poquet $(31,25 \mathrm{~kg} / \mathrm{ha})$ et le mil $1,5 \mathrm{~g} /$ poquet $(46,88 \mathrm{~kg} / \mathrm{ha})$. Ce traitement a été comparé à l'apport à la volée de la dose vulgarisée et à un témoin absolu. La microdose a permis d'augmenter les rendements grains de $143 \%, 134 \%$ et $155 \%$ respectivement pour le sorgho, le mil et le niébé comparativement au témoin. La microdose a été économiquement plus rentable que la dose vulgarisée.

(C) 2017 International Formulae Group. All rights reserved.

Mots clés : Dose vulgarisée, engrais minéraux, microdose, toposéquence, Burkina Faso.

\section{Effects of fertilizer microdosing on cowpea, millet and sorghum production as a function of the toposequence}

\begin{abstract}
The decline in soil fertility has a strong effect on crop productivity and, consequently, on farmers' incomes. The use of mineral fertilizers improves soil productivity, but access is limited to small-scale producers. The objective of this study was to evaluate the agronomic and economic efficiency of the microdose according to the toposequence. Tests were carried out following the toposequence during two cropping seasons and household surveys were conducted to assess the cost-effectiveness of the technology. NPK (14-23-14) fertilizer was applied on cowpea and sorghum at a rate of $2 \mathrm{~g} /$ pit and $3 \mathrm{~g} / \mathrm{pit}$ for millet pocket $(62.5 \mathrm{~kg} / \mathrm{ha})$. Urea was applied only on sorghum and millet respectively at a rate of $1 \mathrm{~g} / \mathrm{pit}(31.25 \mathrm{~kg} / \mathrm{ha})$ and $1.5 \mathrm{~g} / \mathrm{pit}(46.88$ $\mathrm{kg} / \mathrm{ha}$ ). These treatments were compared with the recommended fertilizer rate and an absolute control. Microdose increased grain yields by $143 \%, 134 \%$, and $155 \%$ respectively for sorghum, millet and cowpea compared with the control. Microdose was economically more profitable than the recommended fertilizer rate. (C) 2017 International Formulae Group. All rights reserved.
\end{abstract}

Keywords: Recommended rate, mineral fertilizers, microdose, toposequence, Burkina Faso, 


\section{INTRODUCTION}

La faible productivité des sols constitue un défi majeur pour l'atteinte de la sécurité alimentaire et le développement socio-économique du Burkina Faso. En effet, le secteur agricole emploie près de $90 \%$ de la population active et contribue à près de $40 \%$ du PIB. L'agriculture représente donc un secteur stratégique de développement du pays. Pendant longtemps, l'accroissement de production agricole s'est opéré à travers l'extension des superficies cultivées de fois vers des terres marginales. Cette stratégie extensive a atteint ses limites avec l'explosion démographique qui accentue la pression foncière et risque de compromettre davantage la productivité et la durabilité des systèmes de culture. Cette situation requiert que les producteurs investissent dans l'intensification agricole pour améliorer leur rendement. Or, $72 \%$ des agriculteurs sont des petits producteurs ayant une faible capacité financière et des ressources limitées pour intensifier leur production. En plus, l'accentuation des risques climatiques rendent difficile tout processus d'intensification basé sur la mobilisation du capital financier (Tabo et al., 2005 ; Vall et al., 2011). Il en résulte des conditions de production de plus en plus fragiles et précaires dont les conséquences sont entre autre le faible revenu des populations (Bance, 2013). Des études récentes (Barrett et Bevis, 2015; Vanlauwe et al., 2015) ont montré qu'une faible fertilité des sols maintient les populations dans une pauvreté chronique. De ce fait, pour assurer un développement durable du pays, il est nécessaire d'améliorer la fertilité des sols. Les engrais minéraux sont connus pour leurs effets immédiats et bénéfiques sur les rendements. Pourtant, la majorité des producteurs n'y ont pas accès (Bagayogo et al., 2011) eu égard aux prix élevés, aux difficultés d'accès aux crédits et à l'insuffisance de technologies appropriées pour l'application des engrais minéraux avec pour corollaire un faible taux de fertilisation minérale (8 $\mathrm{kg} / \mathrm{ha})$ et d'adoption des technologies (Bassolé, 2007 ; FAO, 2013). Pourtant, la microdose se pose comme une solution alternative pour pallier ces contraintes. La microdose consiste à des apports de petites quantités d'engrais minéraux dans les poquets (Tabo et al., 2005 ; Taonda et al., 2008 ; Palé et al., 2009 ; FAO, 2012). De nombreuses études ont mis en évidence le rôle positif de la microdose dans la réduction des pertes diverses des nutriments (Demers, 2008), l'amélioration de l'efficience d'utilisation des nutriments par sa concentration au niveau du système racinaire (Tabo et al., 2006 et 2007 ; Palé et al., 2009). Cela se traduit par une croissance rapide des racines et des plantes (Tabo et al.,2005; 2006; 2007 ; Aune et Bationo, 2008; Saba, 2011) l'amélioration des rendements (Tabo et al., 2007 ; Hayashi et al., 2008 ; Taonda et al., 2008 ; Palé et al., 2009 ; Bagayoko et al., 2011; Saba, 2011; Traore et al., 2012; Ibrahim et al., 2015 ; Somda et al., 2017) et du revenu de producteurs (Tabo et al., 2007; Taonda et al., 2008 ; Saba, 2011; Traoré, 2013). Ces auteurs ont rapporté des augmentations de plus de 50\% au Burkina Faso, au Mali et au Niger. Taonda et al., (2008) ont obtenu dans les mêmes régions un $\mathrm{RCV}$, ratio correspondant à la valeur de la production totale/coût des fertilisants, de 2,5 pour le sorgho. Cependant, peu d'études se sont intéressées à l'effet de la toposéquence sur la performance de la microdose. L'objectif de la présente étude était d'évaluer l'efficacité agronomique et économique de la microdose selon la toposéquence.

\section{MATERIEL ET METHODES \\ Description du site d'étude}

L'expérimentation a été conduite durant deux campagnes agricoles 2010-2011 et 2011-2012 dans 10 villages choisis suivant les critères de toposéquence de la commune rurale de Nagréongo $\left(12^{\circ} 28^{\prime} 58^{\prime \prime} \mathrm{N}\right.$ et $1^{\circ} 11^{\prime} 36^{\prime \prime}$ W) région Plateau Central. Située à une 
cinquantaine de kilomètres de Ouagadougou, elle fait partie des sites du projet (AGRAMicrodose) dans lequel ces travaux ont été réalisés. C'est aussi l'un des sites pionniers ayant abrités des tests microdose au Burkina Faso. La commune est dans le climat Nordsoudanien avec une pluviométrie comprise entre 600 et $900 \mathrm{~mm}$ par an. La région est caractérisée par une forte pression démographique $\left(94\right.$ habitants $\left./ \mathrm{km}^{2}\right)$ liée à l'installation de nombreux migrants suite aux Aménagements des Vallées de la Volta (AVV). Il s'en est suivi une forte dégradation des ressources naturelles, la mise en culture des terres marginales et des conflits fonciers (BEM, 2008). La moyenne annuelle des précipitations des 10 dernières années est comprise entre 468 et $860,1 \mathrm{~mm}$. Des pluviosités annuelles de 860,1 et $668 \mathrm{~mm}$ respectivement pour les campagnes agricoles 2010-2011 et 2011-2012.

\section{Caractéristiques des sols}

Les sols ont été caractérisés à travers la description de neuf fosses pédologiques dont trois par niveau de toposéquence. Des échantillons composites sur les horizons 0-20 $\mathrm{cm}$ des parcelles élémentaires et des fosses ont été constitués pour déterminer la teneur en éléments minéraux (texture, matière organique, phosphore assimilable, $\mathrm{pH}$ _eau).

- La texture du sol a été déterminée par la méthode à la pipette Robinson pour déterminer les différentes fractions (argile, limons totaux et sables totaux);

- Le carbone organique a été déterminé par la méthode de Walkley et Black (1934) et la teneur en matière organique déterminée par la formule suivante :

$M O(\%)=[(P i-P f) / P i] \times 100$ et $C(\%)=$ $M O(\%) / 1,724$

Avec : $P i=$ le poids initial de la prise d'essai ; Pf = le poids final de la prise d'essai après calcination.

- Le Phosphore assimilable (P) est extrait selon la méthode Bray I (Bray et Kurtz, 1945) par une solution de fluorure d'ammonium (NH4F) $0,03 \mathrm{M}$ et d'acide chlorhydrique $0,025 \mathrm{M}$ dans un rapport $1 / 7$. Le $\mathrm{P}$ extrait est déterminé par colorimétrie ;

- Le pH a été mesuré par lecture directe en utilisant une électrode électronique dans une suspension de sol diluée à l'eau distillé dans un rapport 1/2,5.

Les résultats de la caractérisation des sols ont montré que les sols expérimentaux de la zone sont à dominance de types ferrugineux tropicaux lessivés, on rencontre aussi des sols bruns et les sols peu évolués en haut de pente. Ces sols sont faiblement acides avec des $\mathrm{pH}$ compris entre 6,0 et 6,2. Leurs teneurs en matière organique et phosphore sont acceptables (Tableau 1).

\section{Le dispositif expérimental et conduite de l'expérimentation}

Les essais ont été conduits en milieu réel durant deux campagnes hivernales. Le dispositif expérimental était un bloc dispersé porté par 15 producteurs pour chacune des trois spéculations (sorgho, mil, niébé) en raison de cinq répétitions par niveau de toposéquence. Les semis ont été réalisés manuellement suivi de deux sarclages et un buttage. Les modes de fertilisation sont constitués du témoin (zéro fertilisation), de l'application à la volée de la dose vulgarisée et la microdose. La microdose a consisté à l'apport du NPK (14-23-14) à la dose de 2 $\mathrm{g} /$ poquet $(62,5 \mathrm{~kg} / \mathrm{ha})$ sur le niébé et le sorgho et $3 \mathrm{~g} /$ poquet $(62,5 \mathrm{~kg} / \mathrm{ha})$ sur le mil à deux semaines après semis. L'urée (46\%) a été apportée à la montaison à la dose de $1 \mathrm{~g}$ $(31,25 \mathrm{~kg} / \mathrm{ha})$ et $1,5 \mathrm{~g} /$ poquet $(46,88 \mathrm{~kg} / \mathrm{ha})$, respectivement pour le sorgho et le mil. Ces différentes doses ont été comparées avec l'application à la volée de la dose vulgarisée $\left(100 \mathrm{~kg} / \mathrm{ha}\right.$ NPK $+50 \mathrm{~kg} \cdot \mathrm{ha}^{-1}$ d'urée) pour le mil et le sorgho et $100 \mathrm{~kg}$ de NPK uniquement pour le niébé et au témoin (zéro fertilisation). Les variétés IKMP5, Kapelga et KVX396-45-2D, respectivement pour le mil, le sorgho et le niébé ont été utilisées dans cette étude. Des 
productions des carrées de rendement ont été extrapolées à l'hectare pour chaque parcelle élémentaire.

La toposéquence est la distribution latérale des structures pédologiques liées à la topographie de la zone (Cirad-Gret, 2002). Dans cette étude, la toposéquence a été appréhendée au niveau micro-bassin c'est-àdire à l'échelle du terroir. Cette considération est liée à l'irrégularité de la pente fortement marquée par de nombreuses zones de partage des eaux délimitant de fait de nombreux micro-bassins à l'intérieur du bassin versant du Nakambé. Trois niveaux de toposéquence, haut de pente, mi-pente et bas de pente, ont été définis à partir d'une carte topographique $\left(1 / 5000^{\text {ieme }}\right)$ comportant le positionnement des parcelles expérimentales.

Détermination de la rentabilité économique La performance économique de la microdose a été évaluée à partir de la rentabilité économique en utilisant le RVC. $\mathrm{Ce}$ ratio permet d'identifier le meilleur traitement qui engendre un rendement additionnel et un gain monétaire optimal. Selon Aune et al. (2007), il correspond au rapport entre la valeur totale de la production et le coût de la fertilisation. Pour Delville (1996), le ratio valeur sur coût est l'indicateur le plus pertinent pour évaluer la rentabilité économique d'une technologie. Il s'obtient par la formule suivante :
Ration Valeur sur Coût (RVC) = Valeur du surplus par rapport au témoin /Coût des fertilisants. Une technologie est économiquement rentable si le RVC $>2$ (Aune et al., 2007). Dans ce cas, le gain obtenu permet au producteur de couvrir les dépenses et de dégager un bénéfice brut car les charges autres que les fertilisants ne sont pas prises en compte. Les informations sur le prix des produits (engrais, denrées) ont été obtenues au moyen d'une enquête réalisée auprès de 40 personnes constituées de producteurs, de commerçants et de prestataires de services agricoles. Les prix utilisés sont celui du marché local au moment des semis pour les engrais soit 17500 FCFA pour NPK et 12500 FCFA pour l'urée le sac de $50 \mathrm{~kg}$. Le coût moyen du kilogramme de grains de sorgho, de mil et de niébé était respectivement, de 125,167 et 283 FCFA contre 19 FCFA pour les tiges de mil et de sorgho et 42 FCFA pour les fans de niébé à la récolte.

\section{Analyses statistiques}

Les analyses statistiques ont été réalisées à l'aide du logiciel Genstat version 7.2. Les données collectées ont été soumises à une analyse de variance (ANOVA) en utilisant le LSD (plus petite différence significative) pour comparer les moyennes des différents traitements au seuil de $5 \%$.

Tableau 1 : Caractéristiques chimiques de l'horizon 0-20 cm des sols expérimentaux.

\begin{tabular}{lcccc}
\hline Toposéquence & MO $\mathbf{\%})$ & $\begin{array}{c}\text { K disponible } \\
(\mathbf{m g} / \mathbf{k g})\end{array}$ & $\begin{array}{c}\text { P assimilable } \\
\mathbf{( m g} / \mathbf{k g})\end{array}$ & pH-eau \\
\hline Haut de pente & 1,0 & 48,5 & $4,50 \mathrm{a}$ & 6,1 \\
Mi-pente & 0,85 & 57,2 & $3,41 \mathrm{~b}$ & 6,0 \\
Bas de pente & 0,96 & 55,2 & $2,54 \mathrm{c}$ & 5,9 \\
Probabilité & 0,318 & 0,580 & $<, 001$ & 0,34 \\
LSD & - & - & 0,755 & - \\
\hline
\end{tabular}

Les moyennes affectées de la même lettre dans une même colonne ne sont pas significativement différentes au seuil de 5\%. 
RESULTATS

Effet des modes de fertilisation sur le rendement du mil, du niébé et du sorgho

Les apports d'engrais ont significativement affecté les rendements grains des différentes spéculations durant les deux campagnes $(p<, 001)$. Les résultats de l'analyse de variance ont montré que durant les deux campagnes, les rendements grains du mil, du sorgho et du niébé sont bien équilibrés entre les traitements microdose et la dose vulgarisée comparativement au témoin (Tableau 2). Le facteur année n'a pas eu d'effet déterminant sur les différents paramètres pris individuellement quelle que soit la spéculation considérée. Notons cependant que l'année 2011 a été légèrement plus élevée que 2010 en raison de la bonne pluviosité enregistrée. La microdose a engendré des accroissements de rendement de $121 \%, 103 \%$ et $140 \%$ en 2010 contre $143 \%$, $134 \%$ et $155 \%$ en 2011 par rapport au témoin respectivement sur le mil, le sorgho et le niébé.
Efficacité de la microdose en fonction de la toposéquence

L'effet combiné de la toposéquence et la fertilisation n'a pas été significatif sur les rendements grains du mil du sorgho et du niébé (Tableau 3). Cependant on note une légère prédominance des traitements dans la partie basse de la toposéquence.

\section{Performance économique de la microdose}

Le Tableau 4 indique que les apports d'engrais permettaient de dégager un gain monétaire supplémentaire pour l'ensemble des cultures. Les apports d'engrais ont permis de dégager un gain monétaire supplémentaire pour l'ensemble des cultures (Tableau 4). Les rapports varient entre 4,3 et 14,8 . Pour l'ensemble des trois spéculations, le RVC est supérieur à 2. Les ratios obtenus par la microdose doublent pratiquement celui de la dose vulgarisée avec la plus grande valeur obtenue par le niébé du fait de sa valeur marchande élevée.

Tableau 2: Effet des modes de fertilisation sur le rendement grain du mil, du sorgho et du niébé.

\begin{tabular}{|c|c|c|c|c|c|c|}
\hline \multirow{2}{*}{ Traitements } & \multicolumn{2}{|c|}{ Mil (kg/ha) } & \multicolumn{2}{|c|}{ Sorgho (kg/ha) } & \multicolumn{2}{|c|}{ Niébé (kg/ha) } \\
\hline & 2010 & 2011 & 2010 & 2011 & 2010 & 2011 \\
\hline Microdose & $1185 \mathrm{a}$ & $1000 \mathrm{a}$ & $1297 \mathrm{a}$ & $1317 \mathrm{a}$ & $1006 \mathrm{a}$ & $1073 \mathrm{a}$ \\
\hline Dose vulgarisée & $1115 \mathrm{a}$ & $966 \mathrm{a}$ & $1220 \mathrm{a}$ & $1234 \mathrm{a}$ & $933 \mathrm{a}$ & 1019 a \\
\hline Témoin & $536 \mathrm{~b}$ & $412 \mathrm{~b}$ & $638 \mathrm{~b}$ & $564 \mathrm{~b}$ & $420 \mathrm{~b}$ & $421 \mathrm{~b}$ \\
\hline Probabilité de F & $<, 001$ & $<, 001$ & $<, 001$ & $<, 001$ & $<, 001$ & $<, 001$ \\
\hline$L S D$ & 109,1 & 84,3 & 116,9 & 107,5 & 118,6 & 76,5 \\
\hline $\mathrm{CV}$ & 15,7 & 14,4 & 15,1 & 14,1 & 20. & 12,4 \\
\hline
\end{tabular}


Tableau 3: Effet de la toposéquence et la fertilisation sur le rendement grain du mil, du sorgho et du niébé.

\begin{tabular}{lcccccc}
\hline Traitements & \multicolumn{2}{c}{ Mil (kg/ha) } & \multicolumn{2}{c}{ Sorgho $(\mathbf{k g} / \mathbf{h a})$} & \multicolumn{2}{c}{ Niébé (kg/ha) } \\
\hline Haut de pente & 2010 & 2011 & 2010 & 2011 & 2010 & 2011 \\
Microdose & 1173 & 948 & 1318 & 1311 & 903 & 1066 \\
Dose vulgarisée & 1036 & 929 & 1231 & 1254 & 851 & 1038 \\
Témoin & 562 & 370 & 613 & 499 & 376 & 411 \\
\hline Mi-pente & 1189 & 1026 & 1254 & 1290 & 989 & 1112 \\
Microdose & 1154 & 954 & 1239 & 1194 & 902 & 1043 \\
Dose vulgarisée & 539 & 440 & 634 & 520 & 411 & 390 \\
Témoin & 1194 & 1026 & 1320 & 1348 & 1126 & 1040 \\
\hline Bas de pente & 1155 & 1015 & 1190 & 1254 & 1045 & 975 \\
Microdose & 506 & 426 & 667 & 674 & 473 & 462 \\
Dose vulgarisée & 0,761 & 0,957 & 0,919 & 0,732 & 0,904 & 0,523 \\
Témoin & - & - & - & - & - & - \\
\hline Probabilité & 16,4 & 14,8 & 16,1 & 14,2 & 19,4 & 12,8 \\
\hline LSD & & & & & & \\
\hline CV & & & & & & \\
\hline
\end{tabular}

Tableau 4 : Effet des traitements sur la rentabilité économique du niébé, du mil et du sorgho.

\begin{tabular}{lcccccc}
\hline \multirow{2}{*}{ Indicateurs économiques } & \multicolumn{2}{c}{ Sorgho } & \multicolumn{2}{c}{ Mil } & \multicolumn{2}{c}{ Niébé } \\
\cline { 2 - 7 } & Microdose & $\begin{array}{c}\text { Dose } \\
\text { vulgarisée }\end{array}$ & Microdose & $\begin{array}{c}\text { Dose } \\
\text { vulgarisée }\end{array}$ & Microdose & $\begin{array}{c}\text { Dose } \\
\text { vulgarisée }\end{array}$ \\
\hline Rendement moyen tige (kg/ha) & 2738 & 2646 & 2380 & 2274 & 712 & 657 \\
Rendement moyen grain (kg/ha) & 1307 & 1227 & 1093 & 1041 & 1040 & 976 \\
$\begin{array}{l}\text { Prix vente de la production tige } \\
\text { (FCFA/kg) }\end{array}$ & 19 & 19 & 19 & 19 & 42 & 42 \\
Coût total de l'engrais & 29687 & 47500 & 33595 & 47500 & 21875 & 35000 \\
RVC & 7,3 & 4,3 & 6,8 & 4,6 & 14,8 & 8,7 \\
\hline
\end{tabular}




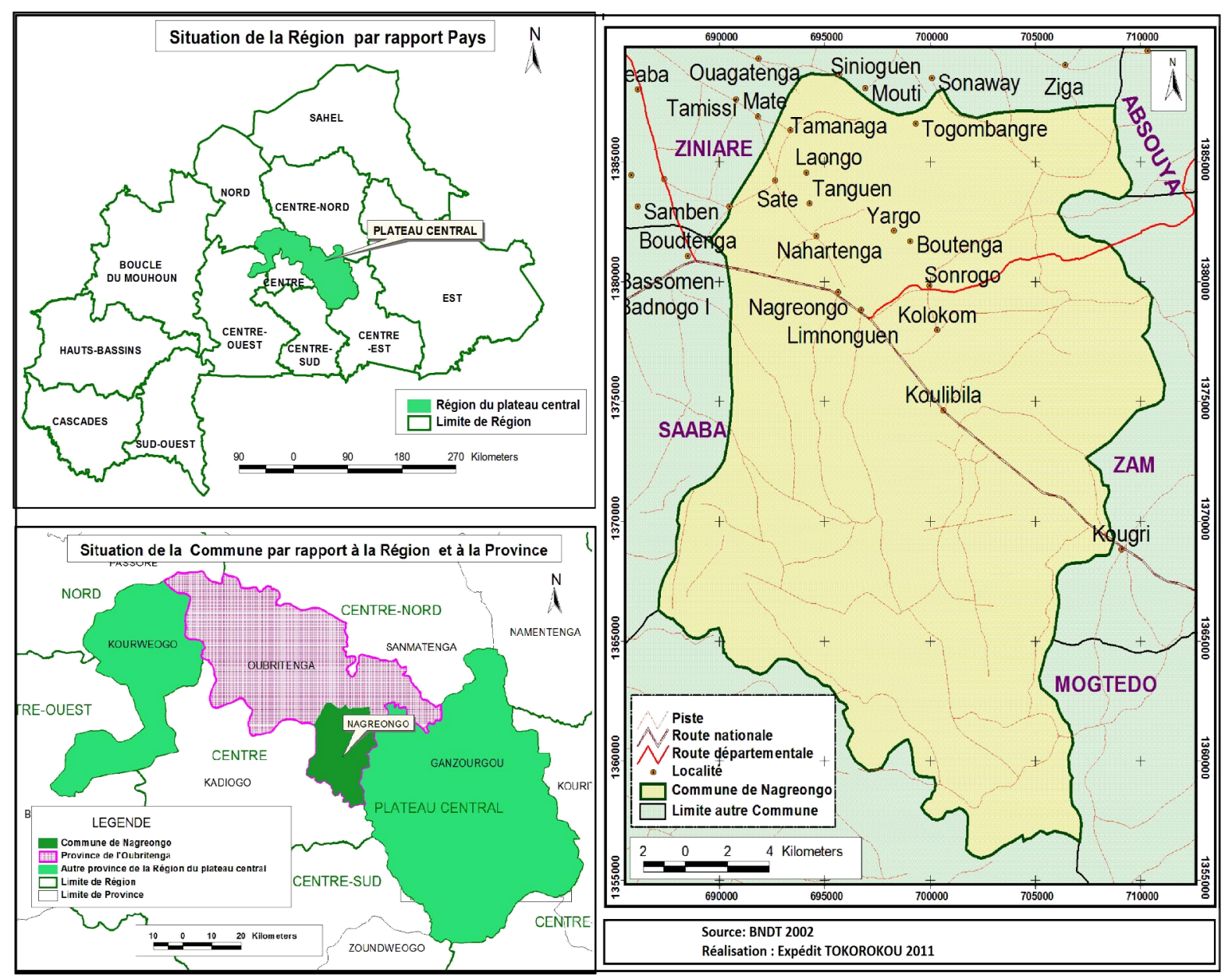

Figure 1 : Localisation de la zone d'étude dans le Burkina Faso et dans la province de l'Oubritenga.

\section{DISCUSSION}

L'accroissement des rendements observé est dû à l'amélioration du statut nutritionnel du sol par les différents apports d'engrais. Ouattara (2007) a montré que la plupart des sols ayant une pauvreté naturelle réagissent positivement aux différentes pratiques d'amélioration de la fertilité. Cette amélioration a engendré une bonne nutrition des plants d'où l'augmentation des rendements. Concernant la microdose, sa performance serait liée à la concentration des nutriments au niveau des systèmes racinaires, ce qui améliorerait l'accessibilité et l'efficience d'utilisation (Muehlig-Versen et al., 2003) et réduirait les pertes. Plusieurs auteurs ont trouvé que la performance de la microdose serait liée au développement précoce des plants (Tabo et al., 2007) ce qui permet aux plantes de maintenir une bonne vigueur dès le départ pour lutter contre les différentes stress. Selon Brück et al., (2003), Hodge (2004), Vadez et al. (2007), et Aune et Bationo (2008), la performance de la microdose s'expliquerait par le fait que la localisation des engrais dans l'horizon superficiel colonisé par les racines des plants engendre leur prolifération et leur croissance ; ceci permet aux plantes de mieux capter les nutriments et l'eau. Nos résultats sont en accord avec ceux obtenus dans la même zone par Agbé (2007), Taonda et al. (2008), et Saba 
(2011). En effet, ces auteurs étaient parvenus à la conclusion que la différence entre la microdose et la dose vulgarisée n'était pas statiquement significative. De même, des études récentes ont montré des résultats similaires avec le mil sur des sols sableux du Niger (Tabo et al., 2007 ; Hayashi et al., 2008; Ibrahim et al., 2014, 2015) ainsi qu'avec le niébé et le sorgho au Mali (Bagayoko et al., 2011) et au Burkina Faso (Taonda et al., 2008 ; Pale et al., 2009).

L'effet combiné de la toposéquence et la fertilisation n'a pas été significatif sur les rendements grain. Ces résultats pourraient s'expliquer d'une part, par la relative bonne pluviométrie des deux campagnes agricoles $(860,1$ et $668 \mathrm{~mm})$ comparativement à la moyenne décennale qui n'ont pas permis à l'effet de la toposéquence de bien s'exprimer et d'autre part, par les caractéristiques des sols des parties hautes de la pente, qui permettaient de conserver l'humidité pour les plantes pendant les éventuelles périodes de déficit hydrique. En effet, les sols des parcelles expérimentales du haut de pente sont majoritairement des sols peu évolués d'apports colluviaux caractérisés par l'horizon de surface gravillonnaire qui repose sur un horizon sous-adjacent argileux; cette structuration permet à ces sols de conserver l'humidité dans l'horizon sous-adjacent pour les plantes favorisant ainsi une bonne nutrition hydrique (Serme et al., 2015). Ces résultats seraient aussi imputable à l'amélioration des conditions du sol (compacité, fertilité et humidité) induite par les différents aménagements antiérosifs (Kagambega et al., 2011) réalisés sur les parties hautes de la pente grâce à l'intervention de plusieurs projets dans la zone.

Sur le plan économique, les résultats intéressants obtenus avec la microdose sont dues au fait que cette technologie permet de réduire les coûts des engrais. Ces résultats sont en accord avec ceux obtenus précédemment, montrant que la microdose favorisait une augmentation du revenu des producteurs (Tabo et al., 2005, 2007 ; Aune et al., 2007 ; Taonda et al., 2008) qui ont montré que la microdose favorisait une augmentation du revenu. Aune et al. (2007) ont obtenu au Mali un RVC variant entre 9,33 et 9,37 pour le sorgho et 3,4 à 11,9 pour le mil avec des apports de 0,3 g/poquet du NPK (15-15-15) ou du DAP (18-46-0). Taonda et al. (2008) ont montré que le revenu est multiplié par 2,5 lorsqu'on passe de dose vulgarisée à la microdose.

\section{Conclusion}

Cette étude a permis d'évaluer la performance de la microdose sur le mil, le sorgho et le niébé. Les apports de microdose d'engrais ont permis d'accroître les rendements de $143 \%, 134 \%$ et $155 \%$ respectivement sur le mil, le sorgho et le niébé par rapport au témoin. Les effets combinés toposéquence et fertilisation n'ont pas été significatifs sur le rendement grain. La microdose est économiquement plus rentable que la dose vulgarisée quelle que soit la spéculation (niébé, sorgho et mil). En somme, la microdose permet en année de pluviosité moyenne de garantir un minimum de rendement quel que soit le niveau de la pente. Cependant des études supplémentaires doivent être faites pour mieux comprendre des facteurs de variabilité de la microdose.

\section{CONFLITS D'INTERETS}

Les auteurs déclarent qu'il n'y a aucun conflit d'intérêts.

\section{CONTRIBUTION DES AUTEURS}

FS a été l'investisseur principal ; SJBT a participé à la conception du dispositif expérimental et l'écriture de l'article. IS et AAB ont participé aux analyses des données et à la correction du projet d'article. AK et APS ont participé au suivi des travaux et à la collecte de données sur le terrain.

\section{REMERCIEMENTS}

Nous adressons nos sincères remerciements à AGRA«Alliance for Green Agriculture in Africa »(AGRA) pour avoir financé cette étude dans le cadre du projet 
microdose ainsi que toutes les personnes ayant contribué sur le plan technique à faire aboutir cette étude.

\section{REFERENCES}

Agbé CO. 2007. Efficacité et efficience de la fertilisation du sol par micro dose de l'engrais NPK selon le type de sol dans le terroir de Nagréongo. Rapport de stage CAP/Matourkou, 46p.

Aune BJ, Bationo A. 2008. Agricultural intensification in the Sahel. The ladder approach. Agricultural Systems, 98: 119$125 . \quad \mathrm{http}: / /$ doi.org/10.1016/j.agsy. 2008.05.002

Aune BJ, Doumbia M, Berthe A. 2007. Microfertilizing sorghum and pear millet in Mali. Agronomic, economic and social feasibility. Agriculture, 36(3): 5. http://journals.sagepub.com/doi/pdf/10.5 367/000000007781891504

Bagayoko M, Maman N, Palé S, Sirifi S, Taonda SJB, Traore S, Mason SC. 2011. Microdose and $\mathrm{N}$ and $\mathrm{P}$ fertilizer application rates for pearl millet in West Africa. African Journal of Agricultural Research, 6(5): 1141-1150. Doi : 10.5897/AJAR10.711

Bance S. 2013. Caractérisation des dispositifs d'accompagnement des Exploitations Agricoles Familiales vers l'intensification durable au Burkina Faso. Memoire Master Agrinovia. 86p

Barrett CB, Bevis LEM. 2015. The selfreinforcing feedback between low soil fertility and chronic poverty. Nature Geoscience $\quad$ 8: $\quad 907-912$ doi:10.1038/ngeo2591

Bassolé D. 2007. Contribution à la réflexion sur la qualité des intrants agricoles au Burkina Faso. Etude sur la qualité des engrais vendus en détails dans 14 localités d'implantation des boutiques d'intrants et de location de matériels au Burkina Faso. APIPAC, IFDC, $39 \mathrm{p}$

BEM. 2008. Plan communal de développement de Nagréongo, 73p

Bray RH, Kurtz LT. 1945. Determination of total, organic, and available forms of phosphorus in soils. Soil Science, 59: 3945.

Brück H, Sattelmacher B, Payne W. 2003. Varietal differences in shoot and rooting parameters of pearl millet on sandy soils in Niger. Plant and Soil, 251: 175. doi:10.1023/A:1022932815486

Cirad-Gret, Ministère des affaires étrangères. 2002. Mémento de l'agronome, 1691p.

Demers I. 2008. Formes et disponibilité du phosphore de composts utilisés comme Amendements de sols agricoles. Mémoire Maître ès Sciences (M.Sc). Faculté des études supérieures/Université de Laval, 92p

Delville PL. 1996. Gérer la fertilité des terres des pays du sahel : diagnostic et conseil aux paysans. Collection le point sur GRET, 397p. http://cataloguebibliotheques.cirad.fr/cgi-bin/koha/opacdetail.pl?biblionumber $=12615$

FAO. 2012. La fertilisation localisée au semis des cultures ou microdose, fiche d'information, Capitalisation des bonnes pratiques en appui à la production agricole et à la sécurité alimentaire, $4 p$

FAO. 2013. Analyse des incitations et pénalisation pour le sorgho au Burkina Faso, Monitoring african food and agricultural policies, suivi des politiques agricoles et alimentaire en Afrique.

Hayashi K, Abdoulaye T, Gerard B, Bationo A. 2008. Evaluation of application timing in fertilizer microdosing technology on millet production in Niger, West Africa. Nutr $\mathrm{Cycl}$ Agroecosyst., $\quad$ 80: 257. doi:10.1007/s10705-007-9141-3

Hodge A. 2004. The plastic plant: root responses to heterogeneous supplies of nutrients. New Phytologist., 162: 9-24. doi: 10.1111/j.1469-8137.2004.01015.x

Ibrahim A, Abaidooa RC, Fatondji D, Opoku A. 2015. Hill placement of manure and fertilizer microdosing improves yield and water use efficiency in the Sahelian low input millet based cropping system. Field Crops Research, 180: 29-36. http://doi.org/10.1016/j.fcr.2015.04.022 
Ibrahim A, Pasternak D, Fatondji D. 2014. Impact of depth of placement of mineral fertilizer microdosing on growth, yield and partial nutrient balance in pearl millet cropping system in the Sahel. Journal of Agricultural Science, 153: 1412-1421. doi: https://doi.org/10.1017/ S0021859614001075

Kagambega FW, Traore S, Thiombiano A, Boussim JI. 2011. Impact de trois techniques de restauration des sols sur la survie et la croissance de trois espèces ligneuses sur les «zipellés » au Burkina Faso. Int. J. Biol. Chem. Sci., 5(3): 901914.

Muehlig-Versen B, Buerkert A, Bationo A, Roemheld V. 2003. Phosphorus placement on acid arenosols of the West African Sahel. Exp. Agric., 39: 307-325.

Palé S, Mason SC, Taonda SJB. 2009. Water and fertilizer influence on yield of grain sorghum varieties produced in Burkina Faso. S.Afr. J. Plant Soil, 26(2): 91-97. doi: $\quad$ http://dx.doi.org/10.1080/ 02571862.2009 .10639939

Ouattara K. 2007. Improved soil and water conservatory managements for cottonmaize rotation system in the western cotton area of Burkina Faso. Doctoral thesis. Swedish University of Agricultural Sciences (SLU), SE90183, Umea, Sweden, $50 \mathrm{p}$.

Saba F. 2011. Performances de la fertilisation par microdose au poquet suivant les niveaux de toposéquence dans le terroir de Nagréongo (Plateau Central). Mémoire ingénieur Institut du Développement Rural/Université Polytechnique de Bobo, $76 \mathrm{p}$.

Serme I, Ouattara K, Taonda JB, Logah V, Pale S, Quansah C, Abaidoo CR. 2015. Impact of tillage and fertility management options on selected soil physical properties and sorghum yield. Int. J. Biol. Chem. Sci., 9(3): 1154-1170. http://dx.doi.org/10.4314/ijbcs. v9i3.2

Somda BB, Ouattara B, Serme I, Pouya BM, Lompo F, Taonda SJB, Sedogo PM. 2017. Détermination des doses optimales de fumures organo-minérales en microdose da ;ns la zone soudanosahélienne du Burkina Faso. Int. J. Biol. Chem. Sci., 11(2): 670-683. http://ajol.info/index.php/ijbcs

Tabo R, Bationo A, Hassane O, Amadou B, Fosu M, Sawadogo-Kabore S, Ndjeunga J, Fatondji D, Korodjouma O, Abdoul A, Koala S. 2008. Fertilizer microdosing for the prosperity of the ressource poor farmers.

Tabo R, Bationo A, Gerard B, Ndjeunga J, Marchal D, Amadou B, Garba MA, Sogodogo D, Taonda JBS, Hassane O, Diallo MK, Koala S. 2007. Improving cereal productivity and farmers income using a strategic application of fertilizers in West Africa. In: Advance in Integrated Soil Fertility Management in Sub-Saharan Africa: Challenges and Opportunities, Bationo A, Waswa B, Kihara J, Kimetu J (eds). Proceeding of Afnet International Symposium 17-21 September 2004, Yaoundé/Cameroun. Springer 2007, 589-598.

Tabo R, Bationo A, Diallo MK, Hassane O, Koala S. 2006. Fertilizer microdosing for the prosperity of small scale farmers in the Sahel: Final report. Global Theme on Agroecosystems Report $N^{\circ} 23$, Niamey, Niger: International Crops Research Institute for the Semi-Arid Tropics, 28p.

Tabo R, Bationo A, Diallo MK, Hassane O, and Koala S. 2005. Fertilizer microdosing for the prosperity of small scale farmers in the Sahel. Final Report 2001-2004, ICRISAT, Niamey, 28p.

Taonda SJB, Yagho E, Soubeiga J, Kabré A. 2008. Projet « Transfert de la technologie de fertilisation microdose et des variétés tolérantes à la sécheresse pour la prospérité des petits producteurs agricoles du Sahel ». Burkina Faso, Rapport final 2005-2008, 66p. 
Traoré A. 2013. Effet de la microdose, des techniques de collecte et gestion des eaux et du warrantage sur le revenu des femmes productrices de niébé dans le Kouritenga et le Zondoma (Burkina Faso). Mémoire de DEA Ecole doctorale pluridisciplinaire/Université de Parakou.

Traore M, Nacro BH, Tabo R, Nikiema A. Ousmane H. 2012. Potential for agronomical enhancement of millet yield via Jatropha curcas oilcake fertilizer amendment using placed application technique. Int. J. Biol. Chem. Sci., 6(2): 808-819, http://ajol.info/index.php/ijbcs

Vadez V, Krishnamurthy L, Kashiwagi J, Kholova J, Devi J, Sharma K, Bhatnagar-Mathur P, Hoisington D, Hash C, Bidinger F. 2007. Exploiting the functionality of root systems for dry, saline and nutrient deficient environments in a changing climate. Journal of SAT Agricultural Research, 4:1-61.

Vall E, Koutou M, Blanchard M, Coulibaly K, Diallo AM, Andrieu N. 2011. Intégration agriculture-élevage et intensification écologique dans les systèmes agrosylvopastoraux de l'Ouest du Burkina Faso (Prov. Tuy).

Vanlauwe B, Six J, Sanginga N, Adesina AA. 2015. Soil fertility decline at the base of rural poverty in sub-Saharan Africa. Nature Plants, 1: 15101 doi:10.1038/nplants.2015.101

Walkley A, Black JA. 1934. An examination of the Detjareff method for determining soil organic matter and a proposed modification of the chromatic acid titration method. Soil Science, 37: 29-38. 